\title{
Magnetic Moment of a Spin, Its Equation of Motion, and Precession
}

\section{OVERVIEW}

The ability to "see" protons using magnetic resonance imaging is predicated on the proton having a mass, a charge, and a nonzero spin. The spin of a particle is analogous to its intrinsic angular momentum. A simple way to explain angular momentum is that when an object rotates (e.g., an ice skater), that action generates an intrinsic angular momentum. If there were no friction in air or of the skates on the ice, the skater would spin forever. This intrinsic angular momentum is, in fact, a vector, not a scalar, and thus spin is also a vector. This intrinsic spin is always present. The direction of a spin vector is usually chosen by the right-hand rule. For example, if the ice skater is spinning from her right to left, then the spin vector is pointing up; the skater is rotating counterclockwise when viewed from the top.

A key property determining the motion of a spin in a magnetic field is its magnetic moment. Once this is known, the motion of the magnetic moment and energy of the moment can be calculated. Actually, the spin of a particle with a charge and a mass leads to a magnetic moment. An intuitive way to understand the magnetic moment is to imagine a current loop lying in a plane (see Figure B1.1.1). If the loop has current $I$ and an enclosed area $A$, then the magnetic moment is simply the product of the current and area (see Equation B1.1.8 in the Technical Discussion), with the direction $\hat{n}$ parallel to the normal direction of the plane. This direction is also determined by the right-hand rule of the current flow. From experiment, the magnetic moment vector $\vec{\mu}$ is found to be proportional to the spin, and the proportionality constant, $\gamma$ (see Equation B1.1.17 in the Technical Discussion), is the gyromagnetic ratio, which is particle-dependent. For the proton, the gyromagnetic ratio is $2.675 \times 10^{8} \mathrm{rad} / \mathrm{sec} / \mathrm{T}$. In many occasions, we use $₹=$ $42.58 \mathrm{MHz} / \mathrm{T}$, which is the gyromagnetic ratio divided by $2 \pi$.

Because the magnetic moment is proportional to the angular momentum, it is obvious that if a particle has no spin, then such a particle will have no magnetic moment. Both ${ }^{16} \mathrm{O}$ and ${ }^{12} \mathrm{C}$ are such elements and thus we cannot image these two nuclei despite their abundance in the human body.

Now we can relate the time derivative of the angular momentum to the torque to derive an equation of motion. In the presence of a magnetic field, the torque is the cross-product of the magnetic moment $\vec{\mu}$ and the magnetic field $\vec{B}$. Because the angular momentum is

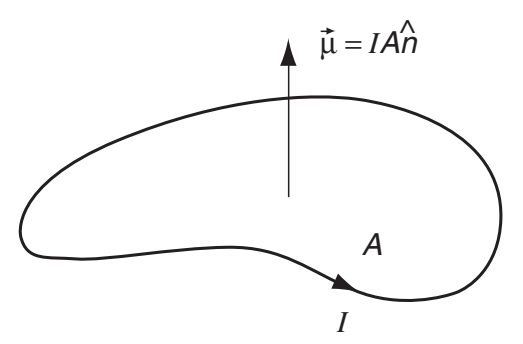

Figure B1.1.1 A loop with current $/$ lying in a plane. The perpendicular unit vector $\hat{n}$ points up from the side where the area $A$ is on our left if we were to walk along the current path in the direction of current flow. The magnetic moment for the loop is I $A \hat{n}$. 
proportional to the magnetic moment, we can write down a first order differential equation of the magnetic moment which describes the motion of a spin:

$$
\frac{d \vec{\mu}}{d t}=\gamma \vec{\mu} \times \vec{B}
$$

This represents part of the famous Bloch equation and basically leads to the description of the precession of a spin in the presence of a magnetic field. Qualitatively, Equation B1.1.1 tells us that the rate of change of $\vec{\mu}$ depends on $\vec{B}$ and that its motion is perpendicular to both $\vec{\mu}$ and $\vec{B}$.

The key to grasping much of MR imaging lies in understanding the concept of precession. Equation B1.1.1 dictates that the spin precesses (rotates) about $\vec{B}$ at the Larmor frequency:

$$
\omega_{\mathrm{L}}=\gamma B
$$

This behavior is shown in Figure B1.1.2, for the magnetic moment $\vec{\mu}$ precessing clockwise about $\vec{B}$. The vector direction of the Larmor frequency points in the direction opposite to that of $\vec{B}$. If the spins start along the $x$-axis, i.e., with a zero phase, then the phase accumulated after a time $t$ is given by:

$$
\phi=-\omega_{\mathrm{L}} t=-\gamma B t
$$

where the minus sign indicates clockwise rotation. Figure B1.1.3 shows the projection of the magnetic moment onto the $x-y$ plane (transverse part of the magnetic moment $\left|\mu_{+}\right|$) and its precession.

Equation B1.1.3 is one of the fundamental equations in MR imaging. The ability to manipulate the phase of a set of spins in a given location (an "isochromat" of spins) will

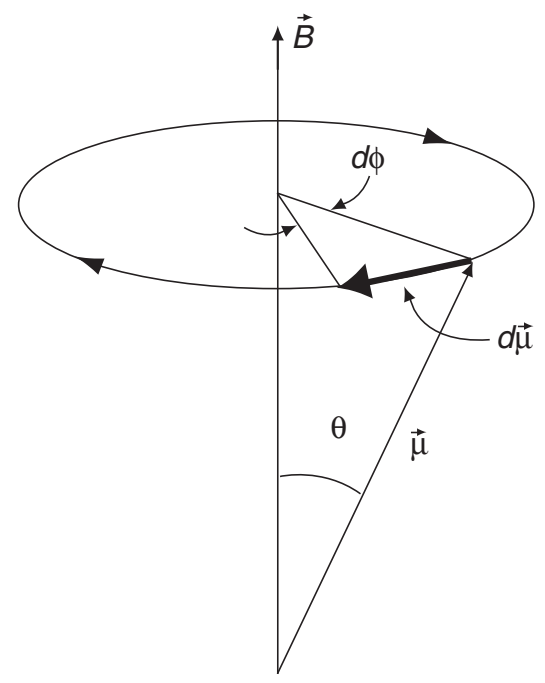

Figure B1.1.2 Clockwise precession of a proton's spin about a magnetic field. As shown, the differential $d \phi$ is negative.

B1.1.2 


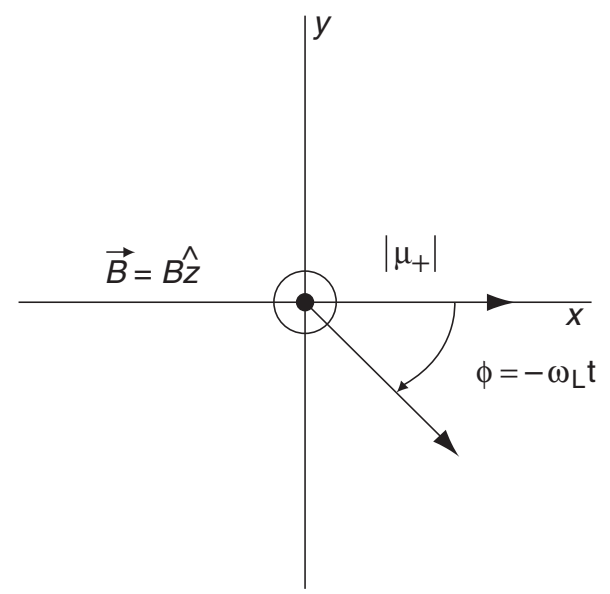

Figure B1.1.3 The transverse part of the magnetic moment from a top view. The magnetic moment precesses through an angle $\phi$ in the $x-y$ plane in a time $t$.

be the backbone of future discussions related to forming echoes, velocity compensating moving spins, and many other processes of importance in designing sequences.

\section{TECHNICAL DISCUSSION}

\section{Torque on a Current Loop in a Magnetic Field}

We begin with a study of magnetic forces on current-carrying conductors, in order to introduce the interaction of a proton with an external magnetic field. A circular loop of current $I$ and area $A$ is pictured with two different orientations in Figure B1.1.4. If an external magnetic field $\vec{B}$ is turned on, the loop will feel a differential force on each of its differential segments $d \vec{\ell}$ given by the basic Lorentz force law:

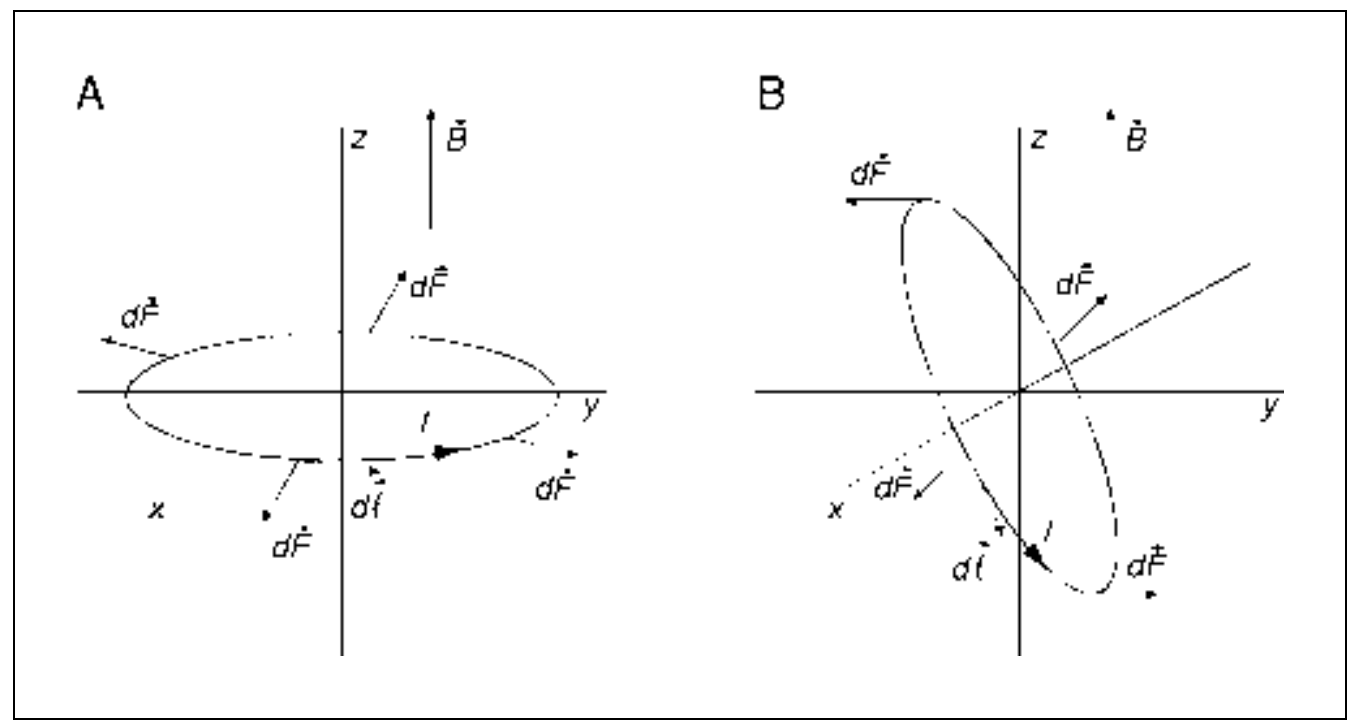

Figure B1.1.4 Circular current loop depicted in two different orientations relative to a uniform magnetic field. The forces on representative differential current segments are shown (one $d \vec{\ell}$ is explicitly shown in each case). The first panel (A) shows the current plane perpendicular to the field where there is no net twist (torque); the second panel $(\mathbf{B})$ shows the current plane at an arbitrary angle to the field where there is a nonzero torque. 


$$
d \vec{F}=l d \vec{\ell} \times \vec{B}
$$

This cross-product implies that the differential force is perpendicular to the plane defined by two vectors: the current segment and the magnetic field evaluated at that segment. It is in the direction that a right-hand screw, perpendicular to the plane, advances when rotated from the current segment vector to the magnetic field vector.

The total force on the circular loop and, indeed, on any closed loop due to a uniform (constant over space) external magnetic field is zero. As an example, the symmetrical vector sum of the differential forces on either current loop in Figure B1.1.4 clearly vanishes for any loop orientation. To prove this, integrate Equation B1.1.4. Both $I$ and $\vec{B}$ can be taken outside the integral. For an integration around any closed path, $\phi \overrightarrow{d \ell}=0$, and this demonstrates that the total force is zero. Zero total force means zero change in the total momentum $\vec{p}$, from Newton's law:

$$
\vec{F}=\frac{d \vec{p}}{d t}
$$

Therefore, a current loop initially at rest in a spatially constant magnetic field stays at rest. For the present, we also assume that the field is constant in time. But this is not the whole story. A current loop can be rotated by the field, depending on the loop's orientation.

Rotations can arise from forces applied off center, even when the vector sum of all forces cancels out. The vector quantity used to describe the rotation of an object is torque $(\vec{N})$. Discussions of torque can be found in most introductory physics textbooks. If the sum of the differential torque contributions:

$$
d \vec{N}=\vec{r} \times d \vec{F}
$$

is nonzero, the current loop can be expected to rotate about the axis along $d \vec{N}$. In Equation B1.1.6, $\vec{r}$ is the position vector from, say, the center of the loop to the point of application of the differential force.

In the special case where the plane of the circular loop is perpendicular to a constant magnetic field, Figure B1.1.4A, each $\vec{r}$ is parallel to its $d \vec{F}$ so each differential torque is zero. Otherwise, when the plane is at some arbitrary angle to that field (Figure B1.1.4B), it is evident that there is a net torque rotating the loop back into the perpendicular plane. We come back to what the proton actually does in response to a similar torque, after discussion of a basic torque formula.

The formula for the net torque on any current distribution, which is exact in a constant magnetic field, is given in terms of the "magnetic dipole moment" or simply "magnetic moment" $\vec{\mu}$ with:

$$
\vec{N}=\vec{\mu} \times \vec{B}
$$

Magnetic Moment of a Spin, Its Equation of Motion, and

Precession

\section{B1.1.4}

with $\vec{\mu}$ itself to be discussed shortly. This cross-product can be taken with respect to any point, but because the net force is zero, the net torque vector is independent of the origin chosen. In place of a general argument for Equation B1.1.7, we present a specific 
calculation of the torque on a circular loop. But first a formula for the magnetic moment of a current loop is needed.

To prescribe the magnetic moment for a planar loop, imagine a right-hand screw perpendicular to the plane of the loop piercing the interior of the loop. The screw is assumed to rotate in the same sense of the current flow. Define a unit vector $\hat{n}$ pointing along the direction in which the screw advances. The magnetic moment vector for planar loops is then given by:

$$
\vec{\mu}=I A \hat{n}
$$

where $A$ is the area of the loop interior. A sample planar moment is illustrated in Figure B1.1.1, along with an alternative description of the right-hand rule.

The torque due to a constant magnetic field is to be calculated for a circular loop with radius $R$ and current $I$ centered in the $x-y$ plane. Let the field lie in the $y-z$ plane and have magnitude $B$. The differential torque on $\overrightarrow{d \ell}$ can be written quite generally as:

$$
d \vec{N}=\vec{r} \times(I d \vec{\ell} \times B)=I d \vec{\ell}(\vec{B} \cdot \vec{r})-I \vec{B}(d \vec{\ell} \cdot \vec{r})
$$

Use has been made of the double cross-product formula after combining Equation B1.1.4 and Equation B1.1.6. The "BAC-CAB" rule is $\vec{A} \times(\vec{B} \times \vec{C})=\vec{B}(\vec{A} \cdot \vec{C})-\vec{C}(\vec{A} \cdot \vec{B})$. The torque can be calculated with respect to the origin (since the net force is zero). The magnetic field and the cylindrical unit vectors shown in Figure B1.1.5 are:

$$
\begin{aligned}
& \vec{B}=B(\cos \theta \hat{z}+\sin \theta \hat{y}) \\
& \hat{\rho}=\cos \phi \hat{x}+\sin \phi \hat{y} \\
& \hat{\phi}=-\sin \phi \hat{x}+\cos \phi \hat{y}
\end{aligned}
$$

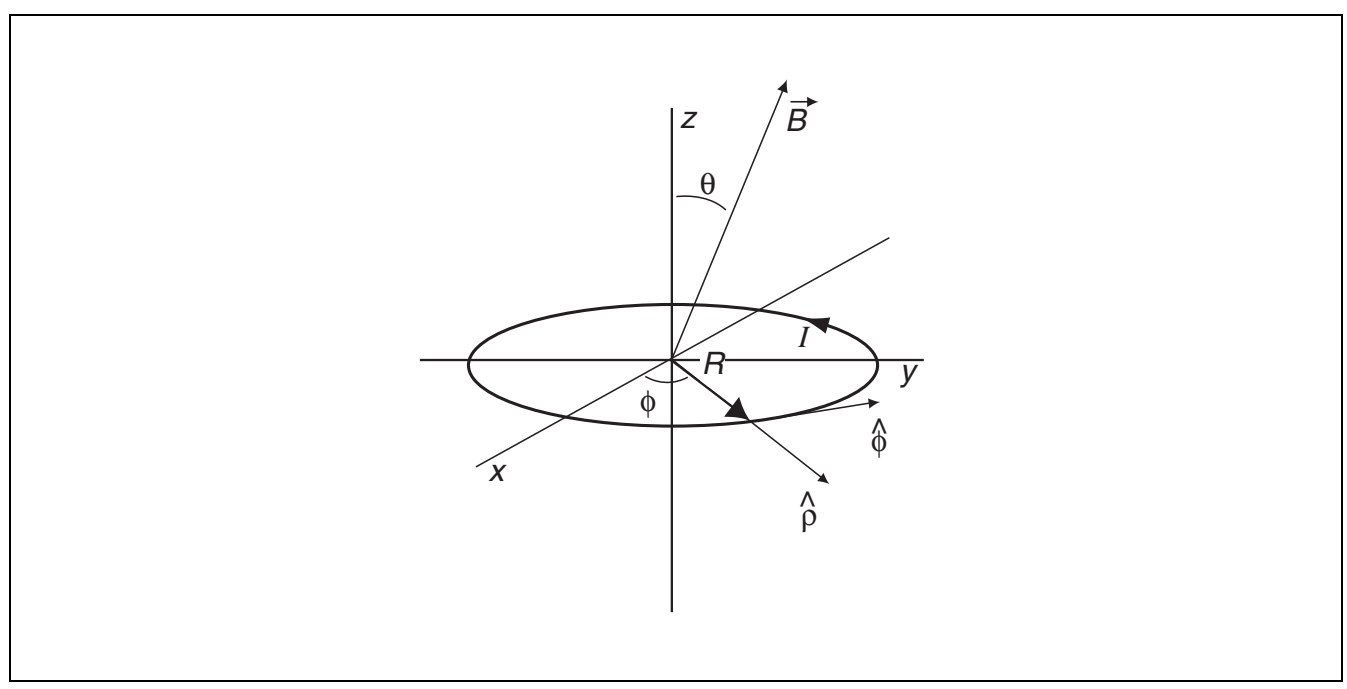

Figure B1.1.5 A circular current loop lying in the $x-y$ plane experiencing a constant magnetic field. The field lies in the $z-y$ plane. 
where the angles are also illustrated in that figure. With $\overrightarrow{d \ell}=R d \phi \hat{\phi}$ and $\vec{r}=R \hat{\rho}$, the second scalar product in Equation B1.1.9 is zero, and a reduction of the first scalar product using Equation B1.1.10 yields:

$$
d \vec{N}=I B R^{2} \sin \theta \sin \phi \hat{\phi} d \phi
$$

An integration of Equation B1.1.11 over the polar angle $\phi$, with $\hat{\phi}$ from Equation B1.1.10, gives the total torque. There is no net $y$-component because $\int_{0}^{2 \pi} d \phi \sin \phi \cos \phi=0$. The integral $\int_{0}^{2 \pi} d \phi \sin ^{2} \phi=\pi$ is needed for the calculation of the net $x$-component, leading to:

$$
\vec{N}=-I \pi R^{2} B \sin \theta \hat{x}
$$

The average value of $\sin ^{2} \phi$ or $\cos ^{2} \phi$ over any multiple of $\pi / 2$ is $1 / 2$. Equation B1.1.12 is exactly $\vec{\mu} \times \vec{B}$ in view of the fact that the magnetic dipole moment for the circular loop of Figure B1.1.5 is:

$$
\vec{\mu}=I \pi R^{2} \hat{z}
$$

Exact for constant fields, the torque formula (Equation B1.1.7) is also very accurate for small loops in a spatially varying field. The only requirement is that the loop scale (say, its diameter $D$ ) must be much less than the typical distances over which the field changes (for example, $|\Delta B| \cong|\partial B / \partial x| D<<|B|$ ). Corrections would arise from, for example, "higher moments" such as electric quadrupole moments. In the case of a proton, however, the electric quadrupole moment, and all other higher moments, are zero.

\section{Torque and Angular Momentum}

The lesson so far is that a magnetic moment, such as that corresponding to a current loop or a bar magnet, will try to line up along the direction of an external magnetic field. This is much as a falling pendulum tries to align itself with the direction of gravity. If the moment is associated with an angular momentum (a "spinning"), then the motion is changed. To see what the new motion is, we introduce the general differential equation for angular momentum in the presence of external torque and the atomic relation between intrinsic angular momenta and magnetic moments.

Nonzero total torque on a system implies that the system's total angular momentum $\vec{J}$ must change according to:

$$
\frac{d \vec{J}}{d t}=\vec{N}
$$

One can derive this equation, which is discussed in most introductory mechanics textbooks. We start with a single point mass case. Consider a point mass $m$ moving at velocity $\vec{v}(t)$ with position $\vec{r}(t)$ defined by some origin. Its angular momentum relative to that origin is therefore $\vec{J}=\vec{r} \times \vec{p}$ with $\vec{p}=m \vec{v}$. Thus, by using Equation B1.1.5 and Equation B1.1.6, 


$$
\begin{aligned}
\frac{d \vec{J}}{d t} & =m \frac{d}{d t}(\vec{r} \times \vec{v})=m \vec{v} \times \vec{v}+m \vec{r} \times \frac{d}{d t} \vec{v} \\
& =\vec{r} \times \frac{d}{d t} \vec{p}=\vec{r} \times \vec{F}=\vec{N}
\end{aligned}
$$

The generality of Equation B1.1.14 follows by considering a system as a limit of many point particles. The total angular momentum is the corresponding limit of:

$$
\vec{J}=\sum_{i} \vec{r}_{i} \times \vec{p}_{i}
$$

with respect to some origin.

\section{Angular Momentum of the Proton}

We next formulate the connection between the proton intrinsic angular momentum (or what is often referred to as its "spin") and its moment. The connections for other nuclear particles are also of interest.

The proton spin can be thought of as leading to a circulating electric current and, hence, an associated magnetic moment. The direct relationship between the magnetic moment and the spin angular momentum vector is found from experiment:

$$
\vec{\mu}=\gamma \vec{J}
$$

The proportionality constant $\gamma$ in Equation B1.1.17 is called the gyromagnetic (or magnetogyric) ratio and depends on the particle or nucleus. For the proton, it is found to be:

$$
\gamma=2.675 \times 10^{8} \mathrm{rad} / \mathrm{sec} / \mathrm{T}
$$

or, what may be referred to as "gamma-bar,"

$$
\gamma=\frac{\gamma}{2 \pi}=42.58 \mathrm{MHz} / \mathrm{T}
$$

where $\mathrm{T}$ represents the Tesla unit of magnetic field and is equal to 10,000 Gauss (G). To this accuracy, the measured value for a proton bound in $\mathrm{H}_{2} \mathrm{O}$ is the same as that for a free proton. Of all the numbers in MR, $₹$ is probably the one most often used in back-of-theenvelope calculations. From Equation B1.1.17 we are justified, in any discussion, to refer either to spin, or to the magnetic dipole moment, since they track each other.

\section{Equation of Motion}

Using both the relationship between the spin and the magnetic moment (via Equation B1.1.17) and Equation B1.1.7 for torque on a magnetic moment due to an external magnetic field $\vec{B}$, we find that Equation B1.1.14 reduces to: 


$$
\frac{d \vec{\mu}}{d t}=\gamma \vec{\mu} \times \vec{B}
$$

This fundamental equation of motion is at the heart of the rotations and precessions that we shall frequently discuss. It is a simple version of the Bloch equation to be presented in Chapter B3. Important corrections arise from the interactions of spins with their surroundings, processes which are referred to as "relaxation" phenomena.

When the time rate of change of a vector is proportional to a cross-product involving that vector, its magnitude $\mu=|\vec{\mu}|$ is unchanged, i.e., $d \mu / d t=0$. This can be seen from the following identity:

$$
2 \mu \frac{d \mu}{d t}=\frac{d \mu^{2}}{d t}=\frac{d}{d t}(\vec{\mu} \cdot \vec{\mu})=2 \vec{\mu} \cdot \frac{d \vec{\mu}}{d t}=2 \vec{\mu} \cdot(\gamma \vec{\mu} \times \vec{B})=0
$$

since $\vec{\mu} \times \vec{B}$ is perpendicular to $\vec{\mu}$. The magnitude may be fixed, but the direction is changing. The instantaneous change in the magnetic moment direction is equivalent to an instantaneous left-handed rotation about $\vec{B}$, the other vector in the cross-product. To see the rotation and get the instantaneous rotation frequency, consider Figure B1.1.2. The differential change in the moment in time $d t$ is $d \vec{\mu}=\gamma \vec{\mu} \times \vec{B} d t$, which is perpendicular to the plane defined by $\vec{\mu}$ and $\vec{B}$. This pushes the tip $\vec{\mu}$ (when viewing from "above" with $\vec{B}$ pointing at the viewer) on a clockwise precession around a circular path. The tip would stay on that same circle if $\vec{B}$ were constant in time. If $d \phi$ is the angle subtended by $d \vec{\mu}$, and $\theta$ is the angle between $\vec{\mu}$ and $\vec{B}$, the geometry of Figure B1.1.2 indicates that:

$$
|d \vec{\mu}|=\mu \sin \theta|d \phi|
$$

On the other hand:

$$
|d \vec{\mu}|=\gamma|\vec{\mu} \times \vec{B}| d t=\gamma \mu B \sin \theta d t
$$

A comparison gives $\gamma B|d t|=|d \phi|$ with $B \equiv|\vec{B}|$, giving the well-known Larmor precession formula:

$$
\omega \equiv\left|\frac{d \phi}{d t}\right|=\gamma B
$$

along an instantaneous axis defined by a left-handed screw rotation about $\vec{B}$. That is:

$$
\frac{d \phi}{d t}=-\omega
$$

Magnetic Moment of a Spin, Its Equation of Motion, and

Precession

\section{B1.1.8}




$$
\vec{\omega}=-\omega \hat{z}
$$

We shall use the convention that angular frequencies are positive. The rotation sense can be indicated by specifying the angular velocity vector, about whose direction the rotation is right-handed. Therefore, the rotation indicated by Equation B1.1.25 is left-handed with respect to the positive $z$-axis.

If the field is along the $z$-axis and constant in time, $\vec{B}=B \hat{z}$, the solution of Equation $B$ 1.1.25 is:

$$
\phi=-\omega t+\phi_{0} \quad \text { (constant field case) }
$$

where $\phi_{0}$ is the initial angle. Again, notice the minus sign ( $\phi$ is the usual azimuthal angle defined in right-handed fashion around the z-axis); Equation B1.1.27 shows constant left-handed precession around the field direction. From now on, we define the Larmor frequency for the constant field case to be:

$$
\omega_{L}(\text { constant field }) \equiv \omega \equiv \gamma B
$$

It is possible to use the rotation picture developed above and trigonometry to derive the behavior of $\vec{\mu}(t)$ for $\vec{B}=B \hat{z}$. In this scenario, the vector differential Equation B1.1.20 decomposes into three Cartesian equations:

$$
\begin{aligned}
& \frac{d \mu_{x}}{d t}=\gamma \mu_{y} B=\omega \mu_{y} \\
& \frac{d \mu_{y}}{d t}=-\gamma \mu_{x} B=-\omega \mu_{x} \\
& \frac{d \mu_{z}}{d t}=0
\end{aligned}
$$

with $\vec{\mu}(t)=\mu_{x}(t) \hat{x}+\mu_{y}(t) \hat{y}+\mu_{z}(t) \hat{z}$ and with time dependence dropped in the above equations. By taking additional derivatives of the first two parts of the above equations, we find:

$$
\begin{aligned}
\frac{d^{2} \mu_{x}}{d t^{2}} & =-\omega^{2} \mu_{x} \\
\frac{d^{2} \mu_{y}}{d t^{2}} & =-\omega^{2} \mu_{y}
\end{aligned}
$$

The solutions for $\mu_{\mathrm{x}}(t)$ and $\mu_{\mathrm{y}}(t)$ are a combination of simple trigonometric functions:

$$
\begin{aligned}
& \mu_{x}(t)=\mu_{x}(0) \cos \omega t+\mu_{y}(0) \sin \omega t \\
& \mu_{y}(t)=\mu_{y}(0) \cos \omega t-\mu_{x}(0) \sin \omega t \\
& \mu_{z}(t)=\mu_{z}(0)
\end{aligned}
$$




\section{Electrons and Other Elements}

It is useful to compare the experimental values for the gyromagnetic ratios with a formula for a simply structured system. Consider a point particle with charge $q$, mass $m$, and speed $v$ traversing in a circle of radius $r$. Recall that the angular momentum $\vec{J}=\vec{r} \times \vec{p}=m r v \hat{n}$ where $\hat{n}$ points in a direction given by the right-hand rule applied to the motion of the particle. The magnetic moment is the product of current and area and its vector direction is apparently parallel to $\hat{n}$. The area enclosed by this point charge is $\pi r^{2}$ and the current is the product of the charge $q$ and frequency, which is the angular frequency divided by $2 \pi$. For a circular motion, the angular frequency is equal to the speed $v$ divided by radius $r$. Putting all these together in Equation B1.1.17 yields the result for the gyromagnetic ratio of a point particle:

$$
\gamma(\text { point charge in circular motion })=\frac{q}{2 m}
$$

This is not an accurate formula for the nuclear particles of interest, but it does help us understand the differences due to mass. For example, choosing the mass $\left(1.67 \times 10^{-27} \mathrm{~kg}\right)$ and charge $\left(1.60 \times 10^{-19} \mathrm{C}\right)$ for a proton, this gives a numerical value of the gyromagnetic ratio of $0.48 \times 10^{8} \mathrm{rad} / \mathrm{sec} / \mathrm{T}$. The difference between this result and Equation B1.1.18 is due to the more complicated motion of the proton constituents, the "quarks." For related reasons, a neutron has a nonvanishing magnetic moment despite its zero overall charge.

From the mass dependence of the previous example, it is not surprising that the $\gamma$ factors can vary from one particle to another, if only because their masses may differ. Indeed, the electron $\gamma$ factor is expected to be much larger than that for the proton in view of the inverse mass dependence. The difference between the observed ratio:

$$
\frac{\left|\gamma_{e}\right|}{\gamma_{p}}=658
$$

and the measured mass ratio $m_{p} / m_{e}=1836$ (the electron mass is $9.11 \times 10^{-31} \mathrm{~kg}$ ) is due to the difference in the structure of the two particles. The electron has no apparent size, while the proton has a size on the order of 1 Fermi $\left(10^{-15} \mathrm{~m}\right)$ and is a complex composite of quarks. They do, however, have exactly the same spin.

Why do we not use electron imaging? The principal reason is the striking difference in the frequency with which a magnetic moment precesses about a static magnetic field. The precession frequency is proportional to the gyromagnetic ratio and, with the difference shown in Equation B1.1.33, it is much larger for the electron. In the standard MRI experiment, an additional, oscillating magnetic field is required; this is the RF field produced by the RF coil whose frequency is matched with the precession frequency. For static fields in the Tesla range, a radiofrequency field in the microwave spectrum is thus needed for electron experiments; however, too much energy would be deposited in human bodies, if electron spins were "excited" by these RF fields. Other problems associated with field inhomogeneities and signal-to-noise, imply that reducing the frequency by reducing the static field strength is not a readily available alternative.

For nuclei, the first requirement is nonzero intrinsic angular momentum (total "spin"). It might be guessed, incorrectly, that magnetic moments of heavier nuclei would be rather small compared to that of a proton, roughly reduced by the inverse ratio of their total masses to the proton mass. In reality, they usually are not very much smaller, nor are they 
ever very much larger. Only the "outer shell" nucleons contribute to the total angular momentum of heavier nuclei; the total nuclear mass is not relevant to the determination of the $\gamma$ factor. In general, protons and, separately, neutrons pair up as much as possible inside of a nucleus, with their spins and orbital motions canceling.

Consider the different nuclear cases. Each "even-even" nucleus (even numbers of protons and even numbers of neutrons) has zero total angular momentum, and, hence, zero magnetic moment. For this reason, we cannot image the ${ }^{16} \mathrm{O}$ and ${ }^{12} \mathrm{C}$ in our bodies with the MR techniques under discussion. The magnetic moment of an even-odd nucleus can be approximately understood in terms of the single unpaired nucleon, but admixtures of states differing in the configurations of the other nucleons must sometimes be taken into account. The unpaired proton and neutron in odd-odd nuclei are not in the same orbital state and do not conspire to give zero spin, in general. For example, nitrogen has twice the spin of hydrogen, and a nonvanishing magnetic moment.

The $\gamma$ factors in Equation B1.1.17 for nuclei with nonzero angular momentum are consequently within an order of magnitude or so of that for the proton. The relation to the nuclear magnetic moment does involve the nuclear spin $I_{N}$ :

$$
\gamma=\frac{\mu}{I_{N}}
$$

The gyromagnetic ratios are determined by measurements, and their values are often rather smaller than that for the proton.

Smaller values for $\gamma$ are not the only reason, however, that imaging of elements other than hydrogen is difficult in the human body. The problem is usually one of low concentration. Still, sodium $\left({ }^{23} \mathrm{Na}\right)$ and phosphorus $\left({ }^{31} \mathrm{P}\right)$ are of imaging interest in view of their nonvanishing magnetic moments (their spins are $" 3 / 2$ " and " $1 / 2$ " in quantum units $\hbar$ in terms of which the proton has spin " $1 / 2$ " where $\hbar$ is defined as $h$ divided by $2 \pi$, where $h$ is Planck's constant, $6.626 \times 10^{-34} \mathrm{~J}$-sec). Their relative $\gamma$ factors, spin, and concentration are listed together with other nuclei of interest in Table B1.1.1.

Table B1.1.1 Spin Properties of Different Elements ${ }^{a}$

\begin{tabular}{lcccc}
\hline Nucleus & Spin & $\begin{array}{c}\text { Magnetic } \\
\text { moment }\end{array}$ & $₹$ & $\begin{array}{c}\text { Abundance in } \\
\text { human body }\end{array}$ \\
\hline Hydrogen ${ }^{1} \mathrm{H}$ & $1 / 2$ & 2.793 & 42.58 & $88 \mathrm{M}$ \\
Sodium ${ }^{23} \mathrm{Na}$ & $3 / 2$ & 2.216 & 11.27 & $80 \mathrm{mM}$ \\
Phosphorus ${ }^{31} \mathrm{P}$ & $1 / 2$ & 1.131 & 17.25 & $75 \mathrm{mM}$ \\
Oxygen ${ }^{17} \mathrm{O}$ & $5 / 2$ & -1.893 & -5.77 & $16 \mathrm{mM}$ \\
Fluorine ${ }^{19} \mathrm{~F}$ & $1 / 2$ & 2.627 & 40.08 & $4 \mu \mathrm{M}$
\end{tabular}

${ }^{a}$ List of selected nuclear species with their spins (in units of $\hbar$ where the proton has spin $1 / 2$ ), their associated magnetic moments in units of a nuclear magneton $\mu_{\mathrm{n}} \equiv 5.05 \times 10^{-27} \mathrm{~A}-\mathrm{m}^{2}$, gyromagnetic ratios $¥$ (in units of $\mathrm{MHz} / \mathrm{T}$ ), and their relative body abundances $(1 \mathrm{M}=1$ molar $=1$ mole/liter). For comparison, the hydrogen $\left({ }^{1} \mathrm{H}\right)$ molarity of water is $110 \mathrm{M}$, and brain gray matter, for example, has a water content of $80 \%$ leading to an abundance of 88 $\mathrm{M}$. The quoted body abundances will vary from tissue to tissue. Certain common elements are omitted, such as ${ }^{12} \mathrm{C}$ and ${ }^{16} \mathrm{O}$, because their nuclear spins (and, hence, their nuclear magnetic moments) are zero. A negative sign for the moment and gyromagnetic ratio refers to the fact that the magnetic moment is anti-parallel to the angular momentum vector. 


\section{KEY REFERENCES}

Beall, P.T., Amtey, S.R., and Kasturi, S.R. 1984. NMR Data Handbook for Biomedical Applications. Pergamon Press, New York.

This text has a more comprehensive list of active NMR elements (i.e., those with nonzero spin).

Goldstein, H. 1980. Classical Mechanics. Addison-Wesley, Reading, Mass.

This text reviews fundamental physics of classical mechanics.

Haacke, E.M., Brown, R.W., Thompson, M.R., and Venkatesan, R. 1999. Magnetic Resonance Imaging: Physical Principles and Sequence Design. John Wiley \& Sons, New York.

This text covers the technical aspects presented here, but in more detail, and also discusses more advanced materials.

Halliday, D., Resnick, R., and Krane, K.S. 1992. Physics. John Wiley \& Sons, New York.

This is a good freshman physics textbook.

Jackson, J.D. 1975. Classical Electrodynamics. John Wiley \& Sons, New York.

This is an advance textbook of classical electromagnetism.

Contributed by Yu-Chung Norman Cheng and E. Mark Haacke

Case Western Reserve University

Cleveland, Ohio and

The MRI Institute for Biomedical Research

St. Louis, Missouri

Magnetic

Moment of a

Spin, Its Equation

of Motion, and

Precession

B1.1.12 